ARTICLE

https://doi.org/10.1038/s41467-019-11476-3

\title{
Maternal control of suspensor programmed cell death via gibberellin signaling
}

Ce Shi ${ }^{1,3}$, Pan Luo ${ }^{1,3}$, Yu-Ting Du ${ }^{1}$, Hong Chen ${ }^{1}$, Xiaorong Huang ${ }^{1}$, Tian-He Cheng ${ }^{1}$, An Luo ${ }^{1}$, Hong-Ju Li (D) ${ }^{2}$, Wei-Cai Yang (ib ${ }^{2}$, Peng Zhao (i) ${ }^{1} \&$ Meng-Xiang Sun ${ }^{1}$

Plant embryos are generated and develop in a stable and well-protected microenvironment surrounded by maternal tissue, which is vital for embryogenesis. However, the signaling mechanisms responsible for maternal tissue-to-proembryo communication are not well understood. Here, we report a pathway for maternal tissue-to-proembryo communication. We identify a DELLA protein, NtCRF1 (NtCYS regulative factor 1), which regulates suspensor programmed cell death (PCD). NtCRF1 can bind to the promoter of NtCYS and regulate the suspensor PCD-switch module NtCYS-NtCP14 in response to gibberellin (GA). We confirm that $G_{4}$, as a primary signal triggering suspensor $P C D$, is generated in the micropylar endothelium by the transient activation of NtGA3oxs in the maternal tissue. Thus, we propose that GA is a maternal-to-proembryo communication signal that is decoded in the proembryo by a GID1-CRF1-CYS-CP14 signaling cascade. Using this mode of communication, maternal tissue precisely controls the embryonic suspensor PCD and is able to nurse the proembryo in a stage-dependent manner.

\footnotetext{
${ }^{1}$ State Key Laboratory of Hybrid Rice, College of Life Sciences, Wuhan University, 430072 Wuhan, China. ${ }^{2}$ State Key Laboratory of Molecular and Developmenta Biology, Institute of Genetics and Developmental Biology, Chinese Academy of Sciences, 100101 Beijing, China. ${ }^{3}$ These authors contributed equally: Ce Shi, Pan Luo. Correspondence and requests for materials should be addressed to P.Z. (email: pzhao2000@whu.edu.cn) or to M.-X.S. (email: mxsun@whu.edu.cn)
} 
B oth animal and plant embryos are generated and developed in a stable and well-protected microenvironment, surrounded by maternal tissue. In animals, the maternal tissue impacts the embryonic development through various signals ${ }^{1-3}$. Recent work on the transcriptome of cells at the maternal-fetal interface in humans has shed new light on the mechanisms underlying maternal-fetal communication ${ }^{4}$. In plants, it is also reported that the maternal tissue, and in particular the seed coat, plays a critical role in embryonic development ${ }^{5-7}$, indicating interaction between maternal tissue and the proembryo is conserved. However, we know few details of the signaling pathways for maternal tissue-to-proembryo communication.

The plant proembryo consists of an embryo-proper domain and a suspensor domain. The suspensor is necessary for embryonic development and plant fertility 8,9 and is functionally similar to the umbilical cord of the mammalian embryo, which transports nutrients and hormones from the mother to the embryo. Thus, the suspensor is the major channel for maternalto-proembryo communication. However, unlike the umbilical cord, which is maintained until birth and provides support throughout embryonic morphogenesis and organ differentiation, the plant suspensor degenerates at a very early stage of embryonic development. This was first discovered over a century ago; however, why and how the suspensor structure degenerates at such an early proembryonic stage is unknown. We previously confirmed that NtCYS-NtCP14 functions as a switch to control the initiation of suspensor $\mathrm{PCD}^{10}$, and this provides a unique opportunity and practical research model for analysis of the maternal-to-proembryo communication via the suspensor.

Here, we identify a tobacco DELLA protein, NtCRF1, and confirm that it is a direct regulator of the suspensor specific PCDswitch NtCYS. We show that transiently increased $\mathrm{GA}_{4}$ in specific maternal tissue functions as a primary signal to trigger GA downstream signaling. NtCRF1 in the suspensor responds to the maternal GA signal to promote timely suspensor PCD. Thus, we reveal a complete GA signaling cascade that mediates the cell-cell communication between maternal tissues and the proembryo, which functions as the key molecular mechanism regulating suspensor PCD.

\section{Results}

NtCRF1 binds to the NtCYS promoter. Based on previous work $^{10}$, we sought to find upstream regulators of NtCYS. We first analyzed the promoter of NtCYS using PlantPan ${ }^{11}$ and used the transcriptome data of two-celled proembryos to select candidate transcription factor (TF)-encoding genes. We screened for candidate TFs that may interact with the NtCYS promoter and selected two homologs NtCRF1 (XP_009779842.1) and NtCRF2 (XP_009789076.1). These both contain GRAS domains that have been reported to bind AATTT motifs ${ }^{12}$. Five AATTT motifs were found in the region $-1.4 \mathrm{~kb}$ upstream of ATG and were named M1-M5 (Fig. 1a), respectively. Using yeast one-hybrid $(\mathrm{Y} 1 \mathrm{H})$ assays, we confirmed that the two TFs stably interacted with the AATTT motifs in the NtCYS promoter (Fig. 1b and Supplementary Fig. 1a). By chromatin immunoprecipitation (ChIP)-qPCR we assayed the five AATTT motifs to verify that NtCRF1 binds to the NtCYS promoter, and identified the motifs that preferentially interact with NtCRF1. In the immature ovaries of a $p N t C R F 1::$ NtCRF1-GFP line, NtCRF1 bound to M3, M4 $\left(P<10^{-4}\right.$, Student's $t$-test), or M5 $(P<0.001$, Student's $t$-test $)$, but not to M1 or M2 $(P>0.05$, Student's $t$-test $)$ in comparison with the control line (pNtCRF1::GFP) (Fig. 1c). We also performed an electrophoretic mobility shift assay (EMSA) to test whether NtCRF1 directly binds to NtCYS promoter in vitro using the potential DNAbinding domain LHRI-VHIID-LHRII, according to a previous report $^{12}$ (Supplementary Fig. 1b). Incubation of the NtCYS promoter probes, including M3-M5, with NtCRF1 LHRI-VHIIDLHRII resulted in a clear band shift (Fig. 1d and Supplementary Fig. 1c, d).

As M3 was the major binding site, we performed a competition assay using unlabeled M3 probe. The binding of NtCRF1 LHRIVHIID-LHRII to M3 was obviously reduced by the unlabeled M3 probe, further confirming the binding between NtCRF1 LHRIVHIID-LHRII and M3. We also mutated the AATTT motif in M3 to GATTT, AGTTT, or CCCCC (Fig. 1e); this greatly reduced the binding of NtCRF1 LHRI-VHIID-LHRII to the promoter fragments (Fig. 1e). A deletion derivative of NtCRF1 (LHRIVHIID-LHRII) bound to M3, but not to the mutants, indicating that the LHRI-VHIID-LHRII domain is responsible for recognition of the AATTT motif (Fig. 1e). NtCRF2 yielded similar results (Supplementary Fig. 1e, f). To test whether NtCRF1 can activate expression of NtCYS, we utilized a dual luciferase assay based on transient transcription in protoplasts of Nicotiana benthamiana (Fig. 1f) and examined the effect of NtCRF1 expression on NtCYS promoter activity. As shown in Fig. 1g, transiently expressed $N t C R F 1$ obviously promoted NtCYS expression in the protoplasts. In addition, we created a mutant $p N t C Y S$, in which the AATTT motif in M3-M5 was replaced by CCCCC. The result confirmed that induction of NtCYS by NtCRF1 depended on the AATTT motif in M3-M5 (Fig. 1f, g).

To gain insight into the spatiotemporal expression pattern of $N t C R F$, we generated transgenic plants carrying the $p N t C R F 1:$ : NtCRF1-GFP or pNtCRF2::NtCRF2-GFP constructs. We observed that NtCRF1 was widely expressed in various tissues (Supplementary Fig. 2). In proembryos, NtCRF1-GFP and NtCRF2-GFP were detected in both basal and apical cell lineages, confirming expression of both NtCRF1-GFP, NtCRF2-GFP and NtCYS$\mathrm{GFP}^{10}$ in the basal suspensor cell (SC) (Fig. 2a and Supplementary Fig. 3a). We also observed that NtCRF1-GFP fluorescence intensity was high in the basal cell lineage $(P>0.05$ compared to stage 1 , Student's $t$-test) until stage 2 , sharply declined at stage 3 $\left(P<10^{-4}\right.$ compared to stage 2 , Student's $t$-test), and was scarcely detectable at stage $4\left(P<10^{-4}\right.$ compared to stage 3 , Student's $t$ test) (Fig. 2b). These changes in expression coincided with those of NtCYS-GFP ${ }^{10}$. Therefore, NtCRF1 may positively regulate the expression of NtCYS in the basal SC.

NtCRF1 regulates NtCYS expression and induces suspensor PCD. To evaluate potential regulatory functions and roles in suspensor PCD, we silenced NtCRF1 using an independent RNA interference (RNAi) construct expressed under the native promoter and selected six homozygous RNAi lines for further analysis (Supplementary Fig. 4a-c). Because NtCRF2 mutants (Supplementary Fig. 3b) showed no seed development phenotypes (Supplementary Fig. 3c, d) and no effects on NtCYS expression (Supplementary Fig. 3e), we subsequently focused on $N t C R F 1$. We analyzed the expression level of NtCYS in 5-DAP seeds (5 days after pollination; Stage 2) to assess the influence of $N t C R F 1$ downregulation on NtCYS expression. The expression level of $N t C Y S$ was significantly reduced in the NtCRF1-RNAi lines (Supplementary Fig. 4c). To exclude any off-target effects of RNAi, we generated Ntcrf1 mutants using the CRISPR/ Cas9 system (Fig. 2c). As expected, the expression level of NtCYS was significantly reduced in the mutants $(P<0.01$, Student's $t$ test) (Fig. 2d). In addition, we determined the expression levels of $N t C Y S$ in NtCRF1 overexpression lines ( $p N t C R F 1:: N t C R F 1-G F P)$ which had significantly greater NtCYS expression $(P<0.01$, Two-way ANOVA) (Fig. 2e), indicating that NtCRF1 promotes the expression of NtCYS and is likely involved in regulating suspensor PCD. 

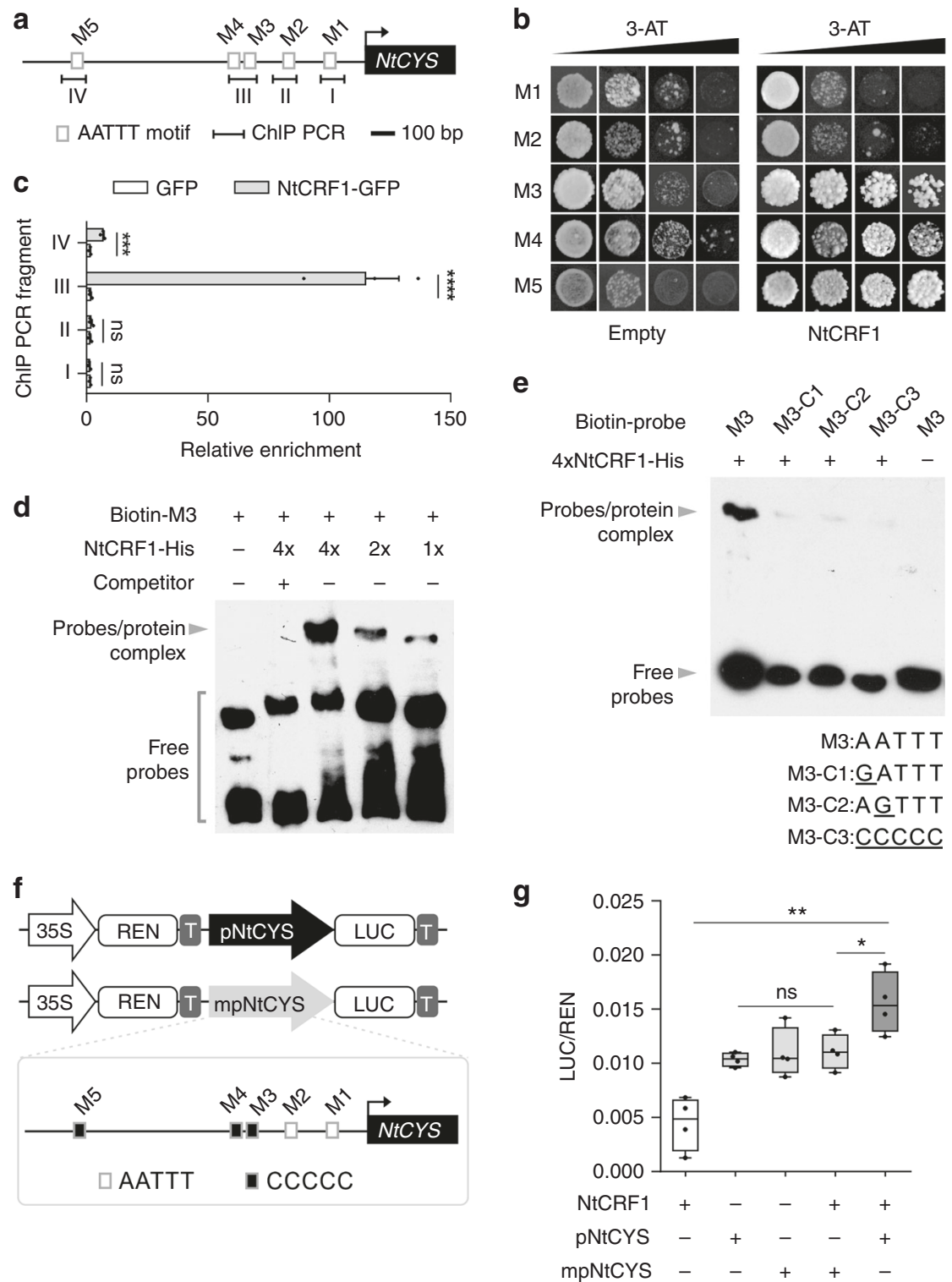

Fig. $1 \mathrm{NtCRF} 1$ binds to the NtCYS promoter. a Distribution of AATTT motifs (M) in the promoter region ( $-1.4 \mathrm{~kb})$ of NtCYS. Fragments I ( -189 to $-83 \mathrm{bp}$ ), II $(-396$ to -296 bp), III ( -609 to -476 bp), and IV ( -1300 to -1211 bp) were subjected to ChIP-qPCR. b Yeast one-hybrid assay of the interaction of NtCRF1 with M3-M5. Empty pGAD424: control. c ChIP-qPCR analysis of the enrichment of NtCRF1 with the four promoter regions. pNtCRF1::GFP, control. Error bars represent the standard error (SE) of three biological replicates. d Binding of recombinant NtCRF1 to M3 was outcompeted by purified NtCRF1 (2 and $4 \times$ ) and biotin-labeled DNA fragments. e Top: Binding of NtCRF1 to M3 mutants was reduced; Bottom: The sequences of M3 and the three mutant motifs. f Structure of the pNtCYS-driven and mpNtCYS (mutant promoter)-driven Dual-Luc reporter gene. 35S promoter (white arrow), pNtCYS (black arrow), mpNtCYS (gray arrow), Renilla luciferase (REN), firefly luciferase (LUC) and terminator (T) are indicated. Box: The mutant motifs of mpNtCYS. g Relative reporter activity (LUC/REN) in tobacco protoplasts. The relative LUC activity was normalized to the REN activity (LUC/REN, $n=4)$. (Student's $t$ test; ns, $P>0.05 ;{ }^{\star} P<0.05 ;{ }^{\star \star} P<0.01$; $\left.{ }^{\star \star \star} P<0.001 ;{ }^{\star \star \star \star} P<0.0001\right)$. The source data of the uncropped immunoblots are provided in the Source Data file

Next, we evaluated nuclear DNA integrity in the early proembryos of wild type (WT), NtCRF1-RNAi, and Ntcrf1 lines using the terminal deoxynucleotidyl transferase dUTP nick-end labeling (TUNEL) technique. In WT plants, the TUNEL signal first appeared in the suspensor at stage 4 (32-celled embryo) ${ }^{10}$. However, in the NtCRF1-RNAi and Ntcrf1 mutant lines, the TUNEL signal was detected at the two-celled proembryo stage (Fig. 2f, g and Supplementary Fig. 4d, e). Moreover, $10-29 \%$ of embryos in NtCYS-RNAi seeds aborted (Supplementary Fig. $4 \mathrm{f}-\mathrm{h}$ ), as did about $28 \%$ of those in Ntcrf1 mutant seeds (Fig. 2h). Notably, the suspensor of the aborted embryos showed abnormal morphology (Fig. 2i and Supplementary Fig. 4f). Due to precocious PCD, the basal cell lineage stopped dividing and degenerated. Thus, these abnormal proembryos were generally without suspensor. As an obvious phenotype, disorganized cell division was observed in all of these abnormal embryos. Pattern formation was disturbed and proembryo development was arrested at early stages. In some of the abnormal proembryos, suspensor structure could still be observed. In fact, although PCD was initiated at stage 1 in most of the proembryos, it could also occur at stage 2 or 3 in the rest of the proembryos. Thus, suspensor-like structures could be still generated in these abnormal proembryos (Supplementary Fig. 4f).

In addition, we also tested the expression of NtCYS by qRTPCR in pNtCRF1::NtCRF1-GFP lines at stage 2 (Fig. 2e). Although the mRNA level of $N t C R F 1$ was increased 

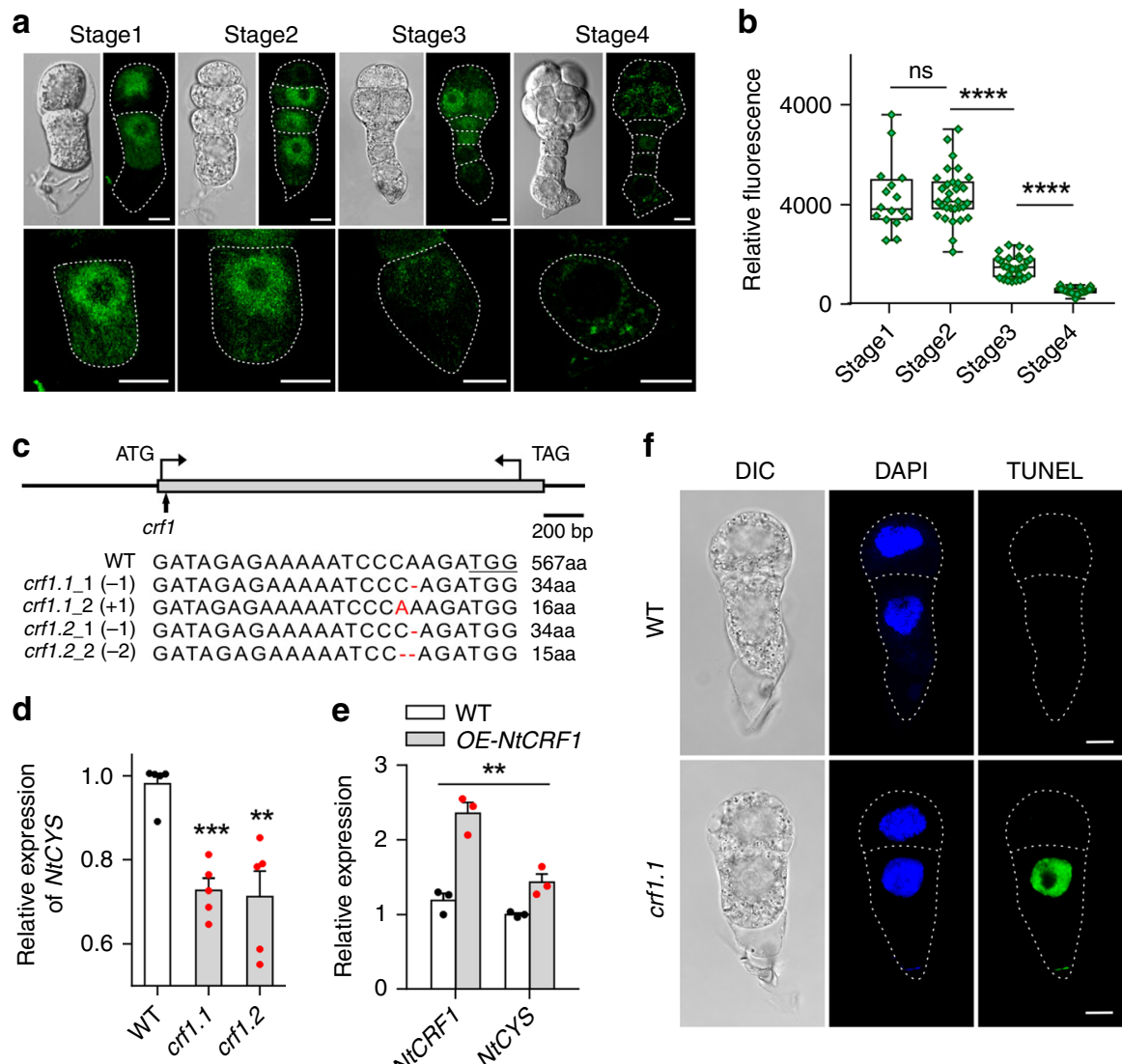

$\mathbf{f}$
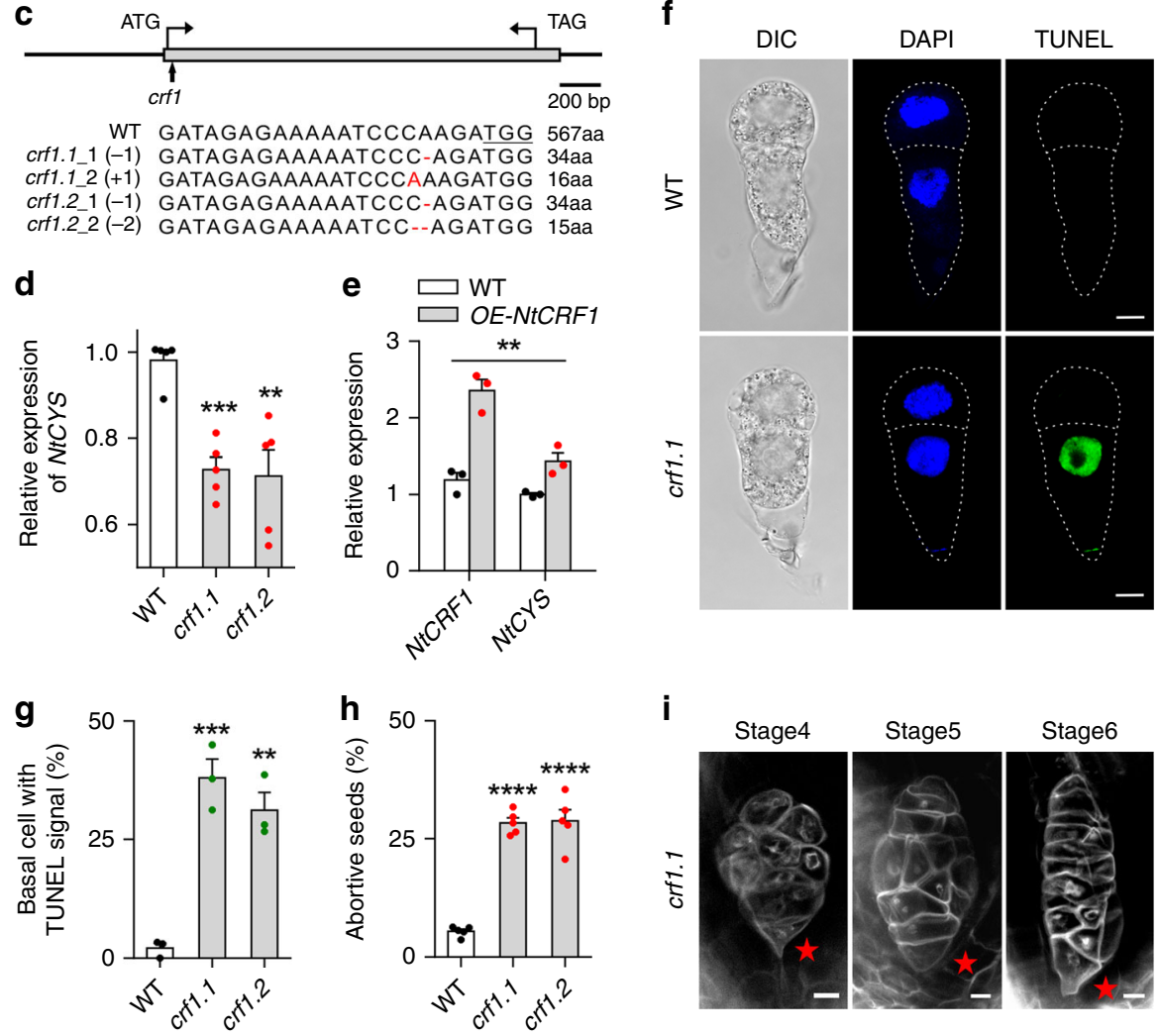

Fig. 2 NtCRF1 promotes NtCYS expression and suppresses PCD in basal cells. a Localization of NtCRF1-GFP during early embryogenesis in pNtCRF1::NtCRF1GFP plants. Magnified images of corresponding basal SCs are shown below. $\mathbf{b}$ Relative fluorescence intensity of NtCRF1-GFP in basal SCs ( $n=16-31$ ). c CRISPR/Cas9-mediated targeted mutagenesis of NtCRF1. Top: Schematic of NtCRF1 showing the CRISPR/Cas9 target site (arrow). Bottom: Alignment of the sequences showing the insertion or deletion sites (red); numbers, translated amino acids (aa). $\mathbf{d}$ Expression of NtCYS was reduced in the crf1 mutants. e Expression of NtCYS is increased in the pNtCRF1::NtCRF1-GFP line (OE-NtCRF1). (Two-way ANOVA, ${ }^{\star \star} P<0.01$ ). $\mathbf{f}$ Nuclear DNA fragmentation in twocelled proembryos of WT and crf1.1. $\mathbf{g}$ Frequency of two-celled proembryos with TUNEL-positive basal cells in WT and crf1 ( $n=98-109)$. $\mathbf{h}$ Frequency of aborted seeds in WT and crf1 lines ( $n=200-300$ seeds per line). i Abnormal suspensors (stars indicated) in crff lines by modified pseudo-Schiff-propidium iodide (PI) staining. Data are the means \pm SE of 3 independent experiments in $\mathbf{e}, \mathbf{g}$ and 5 independent experiments in $\mathbf{d}, \mathbf{h}$ (Student's $t$-test, ${ }^{\star} P<0.05,{ }^{\star \star} P<$ $\left.0.01,{ }^{\star \star \star} P<0.001\right)$. Scale bars: $10 \mu \mathrm{m}(\mathbf{a}, \mathbf{f}), 20 \mu \mathrm{m}(\mathbf{i})$. The source data of the graphs are provided in the Source Data file

(Supplementary Fig. 5a), NtCRF1-GFP soon disappeared at stage 4 (Fig. 2a). In this case, the expression of NtCYS showed no notable change at stage 4 (Supplementary Fig. 5a) and thus, the PCD initiation occurs normally as in WT (Supplementary Fig. 5b, c). These data suggest that NtCRF1 suppresses PCD by promoting $N t C Y S$ expression during normal suspensor development. This anti-cell-death role of NtCRF1 is indispensable for the formation of a functional suspensor and normal embryogenesis.

NtCRF1 functions as a DELLA protein in early embryogenesis. Sequence analysis suggests that NtCRF1 is a member of the DELLA family (Supplementary Fig. 6). Most DELLA proteins can bind to the GA receptor GIBBERELLIN-INSENSITIVE DWARF
1 (GID1) in the presence of bioactive $\mathrm{GA}^{13}$. After binding to GAGID1, DELLA proteins are rapidly degraded ${ }^{14}$.

To determine whether NtCRF1 responds to GA, we examined NtCRF1-GFP fluorescence in the basal cell of two-celled proembryos of the $p N t C R F 1:: N t C R F 1-G F P$ line. After $\mathrm{GA}_{4}$ treatment, NtCRF1-GFP fluorescence was significantly reduced (Fig. 3a, b), indicating the degradation of NtCRF1. Furthermore, treatment of 4-DAP seeds of $p N t C R F 1:: N t C R F 1-G F P$ line with the GA biosynthesis inhibitor paclobutrazol (PAC) resulted in the maintenance of the NtCRF1-GFP signal until stage 5, whereas in control, the NtCRF1-GFP signal was not detected at this stage (Fig. 3c). These data suggest that NtCRF1 responds to GA and may play a role in GA signaling during suspensor PCD. 

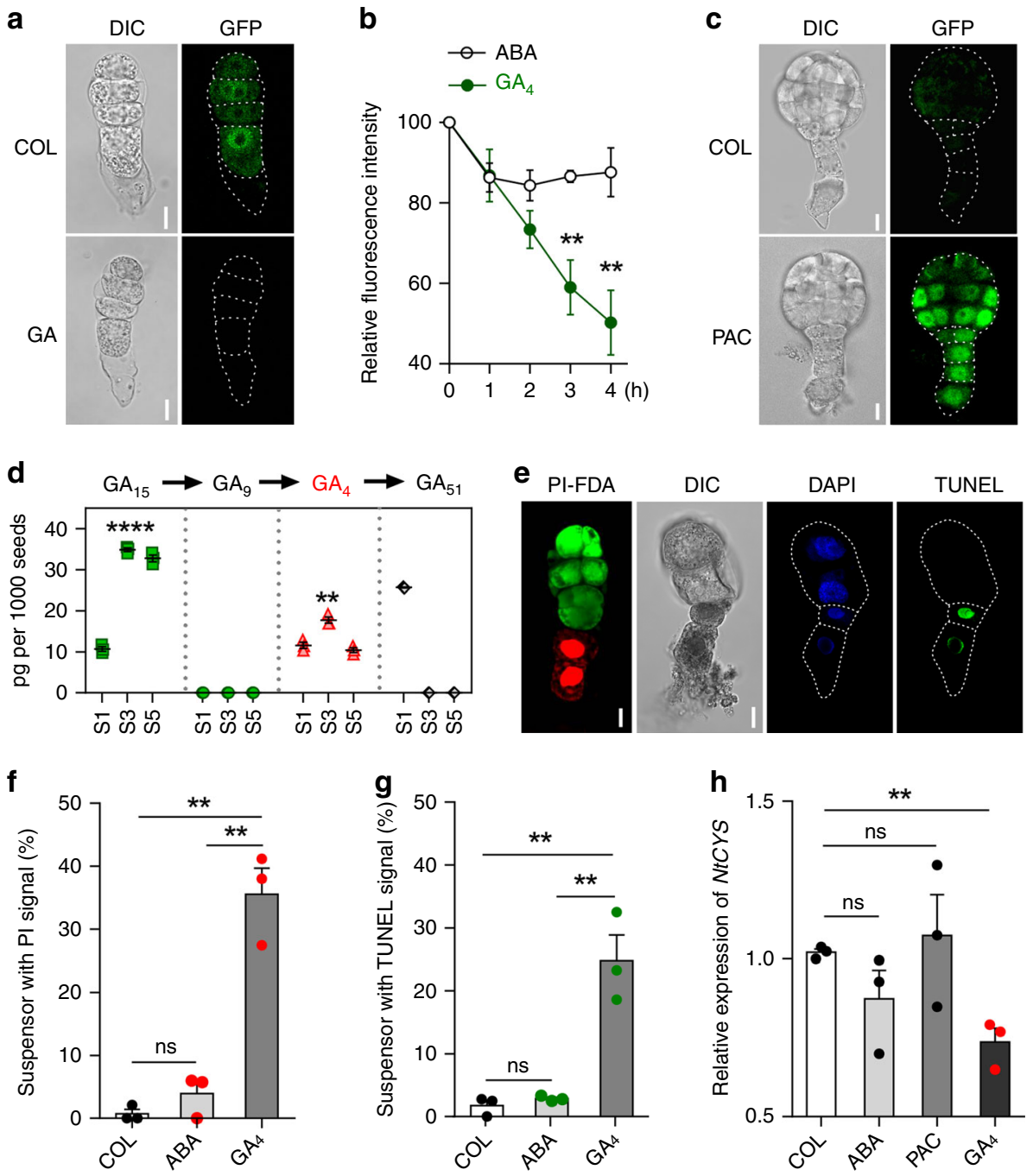

Fig. 3 GA induces suspensor PCD via the NtCRF1-NtCYS pathway in vitro. a NtCRF1-GFP fluorescence in response to GA $A_{4}$ in four-celled proembryos. $0.05 \%$ ethyl alcohol as control. b NtCRF1-GFP fluorescence in the basal cells of two-celled proembryos during treatment with $\mathrm{GA}_{4}$ (or $\mathrm{ABA}$ ) $(n=9)$. c NtCRF1-GFP in embryos derived from two-celled proembryo in cultured seeds after 72-h treatment with PAC. d Quantification of GA $15, \mathrm{GA}_{9}$, GA 4 , and $\mathrm{GA}_{51}$ in WT seeds at stages 1, 3, and 5 (pg per 1000 seeds, $\pm \mathrm{SE}$ ). e Cultured embryos stained with fluorescein diacetate (FDA), PI, and TUNEL. f Frequency of cultured embryos with PI-positive basal cells $(n=128-152)$. $\mathbf{g}$ Frequency of treated cultured embryos with TUNEL-positive basal cells $(n=106-126)$. h qRT-PCR analysis of NtCYS expression in 4-DAP seeds after treatment for 12-h with $100 \mu \mathrm{M} \mathrm{ABA}, \mathrm{PAC}$, or GA 4 . Data are the means \pm SE of three independent experiments (Student's $t$-test; $\mathrm{ns}, P>0.05 ;{ }^{\star \star} P<0.01 ;{ }^{\star \star \star \star} P<0.0001$ ). Scale bars: $10 \mu \mathrm{m}$. The source data of the graphs are provided in the Source Data file

To confirm the role of NtCRF1 in GA signaling, we found all seven $N t G I D 1$ genes in tobacco genome ${ }^{15}$ (Supplementary Fig. $7 \mathrm{a}$ ). We found evidence that four were expressed in two-celled proembryos (Supplementary Fig. 7b). NtGID1B and NtGID1B2 were not found in the two-celled promembryo RNA-seq dataset. Next, by yeast two-hybrid (Y2H) assay we confirmed that, in the presence of bioactive GA, NtCRF1 interacts with NtGID1B3 (XP_016477564.1), NtGID1B5 (XP_016489970.1), and NtGID1C2 (XP_016494194.1) GA receptors, respectively (Supplementary Fig. 7c). Furthermore, these three NtGID1s were expressed in the suspensor of a 32-celled embryo, the stage at which suspensor PCD is triggered (Supplementary Fig. 7d).

To further confirm that NtCRF1 functions as a DELLA protein, we observed seedling development in both the NtCRF1 overexpression line L8 (p35S::NtCRF1-GFP) (Supplementary Fig. 8a) and Ntcrf1.1 mutant. We found that L8 showed shorter hypocotyls (Supplementary Fig. 8b, c), whereas Ntcrf1.1 showed longer hypocotyls. The phenotype of hypocotyl was attributed to a change in cell length, rather than cell number (Supplementary Fig. 8d-f). Furthermore, we demonstrated that these plants have altered responses to GA and PAC treatment, indicating a role of NtCRF1 in GA-induced hypocotyl growth, similar to that of known DELLAs (Supplementary Fig. 8g-j). In addition, we expressed NtCRF1-GFP and (crf1- $\Delta 17)$-GFP, without the DELLA domain, in tobacco BY-2 cells. We found that the GFP fluorescence in the nuclei of the BY-2 cells expressing (crf1$\Delta 17)$-GFP was not affected by GA treatment, whereas nuclear fluorescence was not detectable in the BY-2 cells expressing NtCRF1-GFP after the same GA treatment (Supplementary Fig. 8k). Therefore, these data confirm that NtCRF1 functions as a DELLA protein and participates in the GA signaling pathway during early embryogenesis.

GA as a primary signal induces suspensor PCD. Based on above results we hypothesized that GA is a primary signal that triggers suspensor PCD via NtCRF1. Therefore, we first investigated 
whether endogenous bioactive $\mathrm{GA}_{1}$ or $\mathrm{GA}_{4}$ are present in WT seeds at stage 1,3 (prior to suspensor PCD), and 5 by ultraperformance liquid chromatography tandem mass spectrometry (UPLC-MS/MS). We detected $\mathrm{GA}_{4}$, but not $\mathrm{GA}_{1}$, before suspensor PCD (Fig. 3d and Supplementary Fig. 9a), suggesting that $\mathrm{GA}_{4}$ triggers suspensor PCD. Then, using our embryo culture system ${ }^{16}$, we tested whether $\mathrm{GA}_{4}$ is capable of inducing suspensor $\mathrm{PCD}$. We cultured two-celled proembryo cells with exogenous $\mathrm{GA}_{4}$ or abscisic acid (ABA; control). Approximately $36 \%$ of the cultured proembryos had propidium iodide (PI)-positive and fluorescein diacetate (FDA)-negative SCs after 72-h (stage 3) $(P<$ 0.01 compared to ABA or $0.05 \%$ ethyl alcohol, Student's $t$-test), indicating that $\mathrm{GA}_{4}$ induces SC death (Fig. 3e, f). To confirm this finding, we evaluated nuclear DNA integrity in $\mathrm{GA}_{4}$-treated proembryos by TUNEL within 48 -h (stage 2 ). In addition, the NtCYS expression was also decreased within 12-h (Fig. 3h) $(P<$ 0.01 compared to $0.05 \%$ ethyl alcohol, Student's $t$-test). The suspensors of $25 \%$ of proembryos were TUNEL-positive (Fig. $3 \mathrm{e}$, g). We further tested the suspensor PCD when GA biosynthesis was inhibited by PAC treatment at stage 4 . We found that the PCD delayed and the suspensor cells could continue dividing at the stage (Supplementary Fig. 10). Therefore, it is clear that $\mathrm{GA}_{4}$ as a signal induces suspensor PCD via the NtCRF1-NtCYS pathway.

To examine how the timing of bioactive GA accumulation may be controlled, we investigated the dynamic distribution of bioactive GA. GA3ox, a GA oxidase, catalyzes the final step of the pathway to produce $\mathrm{GA}_{1}$ and $\mathrm{GA}_{4}$. Its activity is therefore indicative of GA synthesis and distribution ${ }^{17}$. Thus, we cloned all homologous GA3ox genes expressed in seeds before suspensor PCD, including the known NtGA3ox1 (AB032198) and NtGA3ox2 (EF471116) genes, and a homologous GA3ox gene, named NtGA3ox3 (XP_016453191.1) (Supplementary Fig. 11). We examined the expression of these NtGA3oxs in seeds. Interestingly, expression of the three $N t G A 3 o x$ genes peaked prior to initiation of suspensor PCD (stage 4) (Fig. 4a) and remained at a low level thereafter. This expression pattern of key bioactive GA synthesis genes suggests a transient increase in the level of bioactive GA in seeds immediately before stage 4, which may trigger PCD of SCs. To confirm the above, we used nlsGPS1 [a nucleus-targeted variant of gibberellin perception sensor 1 (GPS1)] as a bioactive GA sensor in vivo ${ }^{18}$. We generated stable transgenic lines expressing $p$ L25::nlsGPS1. L25 was demonstrated as a reference gene with high expression stability ${ }^{19}$ and wide expression in vegetative tissues and seed including seed coat, endosperm, and embryo. We assessed the relative bioactive GA content by calculating the nlsGPS1 emission ratios. The results showed that the level of bioactive GA was significantly increased $\left(P<10^{-4}\right.$, Student's $t$-test $)$ at stage 3 compared to that at stage 1 (Fig. 5a, b), in agreement with the NtGA3oxs expression (Fig. 4a) and $\mathrm{GA}_{4}$ content data (Fig. 3d). Thus, immediately before suspensor PCD, NtGA3oxs expression is upregulated, and $\mathrm{GA}_{4}$ is therefore transiently increased, which degrades NtCRF1 and triggers suspensor PCD.

Maternal GA triggers suspensor PCD. We next sought to investigate the specific tissue that is the source of $\mathrm{GA}_{4}$ that triggers suspensor PCD. Because PCD occurs in basal SCs, we inferred that GA may be produced in the embryo, the surrounding endosperm cells, or the maternal tissue (i.e., the seed coat) and move to the basal SC. In two-celled and four-celled proembryos, genes related to GA signaling were expressed, but those associated with GA biosynthesis and metabolism were not or at a quite low level (Fig. 4b). Next, we generated three $p N t G A 3 o x:: H 2 B-G F P$ constructs to determine the location of GA synthesis. NtGA3ox1, NtGA3ox2, and NtGA3ox3 were not expressed in the endosperm and proembryo of the transgenic lines before stage 3 (Fig. 4c, d and Supplementary Fig. 9c, d). Furthermore, the three NtGA3oxs were not expressed in the suspensor of a 32-celled embryo, the stage at which suspensor PCD is triggered (Supplementary Fig. 9b). However, these genes were expressed in the seed coat and towards the micropyle region up to stage 4 (Fig. 4d and Supplementary Fig. 9d). This suggests that the $\mathrm{GA}_{4}$ that triggers PCD likely originates from maternal tissue, particularly the micropyle part of the seed coat.

Accordingly, we found that bioactive GA exhibited a polar distribution in developing seeds: the mean nlsGPS1 emission ratio increased significantly from the chalaza to the micropylar ends $\left(P<10^{-4}\right.$, Student's $t$-test) (Fig. 5a, c). This is in agreement with the polar activation of NtGA3oxs at the same stage (Fig. $4 \mathrm{~d}$ and Supplementary Fig. 9d). Furthermore, we found that bioactive GA was localized mainly to the inner layer of the seed coat, the endothelium. The bioactive GA signal arose in the micropyle pole of the endothelial layer (Fig. 5a, d), to which the suspensor is connected. Therefore, it is clear that the bioactive GA level is transiently increased in the micropylar endothelium cells adjacent to the basal SC due to timely activation of NtGA3oxs.

Thus, maternal GA likely enters the basal SC and induces the GA response. To confirm this, we analyzed active GA signaling in the pNtCRF1::NtCRF1-GFP line. Before stage 2, we observed a strong GFP signal in the basal SC, which rapidly diminished as the GA level in the micropyle endothelium increased (Fig. 5e), indicating activation of GA signaling in the basal SC. Interestingly, the GFP signal gradually declined from the basal SC to the vertically adjacent cell (Fig. 5e), as did the occurrence of PCD. The nlsGPS1 emission ratio indicated that bioactive GA appeared in the basal SC at stages 2 and 3 (Fig. 5f, g), and subsequently moved to the vertically adjacent cell along with a decrease in the NtCRF1-GFP signal. This is in consistent with sequential decline of NtCRF1-GFP and occurrence of PCD. Therefore, entry to the basal cell of maternal GA triggers suspensor PCD.

To further confirm the induction of suspensor PCD by maternal GA in vivo, we ectopically expressed NtGA3ox1 in the endothelium, endosperm, proembryo, and seed coat under the control of four tissue-specific promoters, respectively (Fig. 6a and Supplementary Fig. 12a). Ectopic expression of NtGA3ox1-GFP in the seed coat, endosperm, and proembryo did not influence $N t C Y S$ expression (Supplementary Fig. 12 b-d). However, ectopic expression of NtGA3ox1-GFP in the endothelium, to which the basal SC is connected, downregulated NtCYS expression (Fig. 6b). We also evaluated proembryo development in the pTPE8:: NtGA3ox1-GFP (TG) lines. As expected, we detected PCD at the two-celled proembryo stage, as in the NtCRF1 downregulation and NtCYS-RNAi lines. The basal cells in approximately $25 \%$ of the two-celled proembryos contained TUNEL-positive nuclei (type 1), as did both cell types of around $57 \%$ of the two-celled proembryos (type 2) (Fig. 6c, d). This basal cell PCD prevents suspensor establishment during embryogenesis (Fig. 6f), resulting in the abortion of about $50 \%$ of seeds (Fig. 6e). Next, we crossed the $p N t C R F 1:: N t C R F 1-G F P$ line with the TG-8 line to monitor the dynamics of NtCRF1 in the presence of ectopic expression of NtGA3ox1-GFP in the endothelium. As expected, NtCRF1-GFP fluorescence was absent from the four-celled and two-celled proembryos (13/24) (Fig. 6g). Therefore, suspensor PCD is triggered, via NtCRF1, by bioactive GA from maternal endothelial cells.

In addition, we also tried to look at the relevant aspects in Arabidopsis. As reported, AtGA3oxs are inactive during early embryogenesis $^{17}$. Transcriptome analysis of GA3oxs and GA20oxs also suggests that GA biosynthesis is inactive in early proembryos $^{20}$. GA signaling activation after fertilization specifically 
a

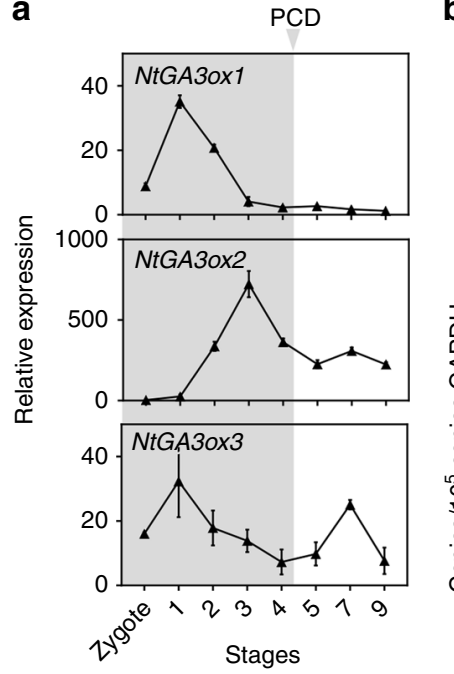

b

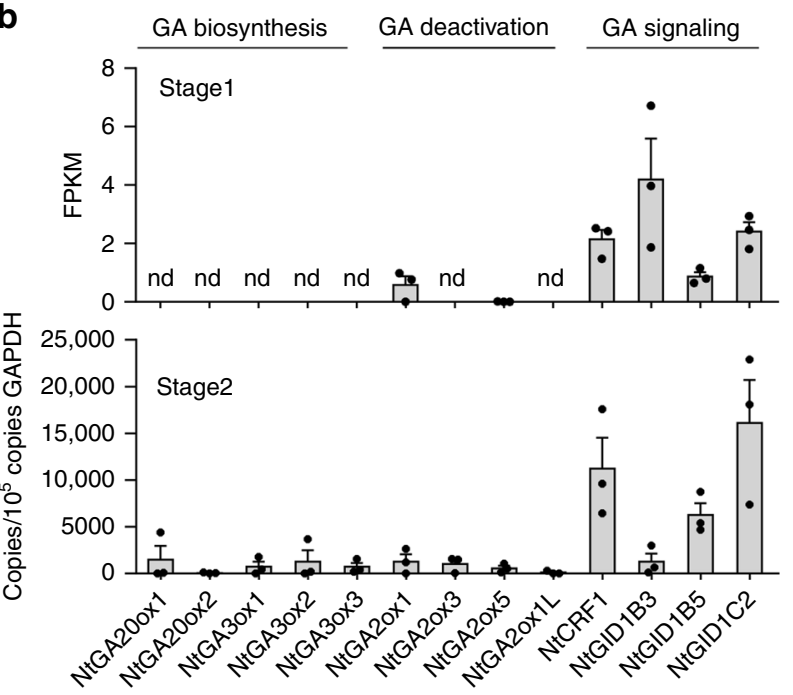

C $p N t G A 30 \times 1:: H 2 B-G F P$
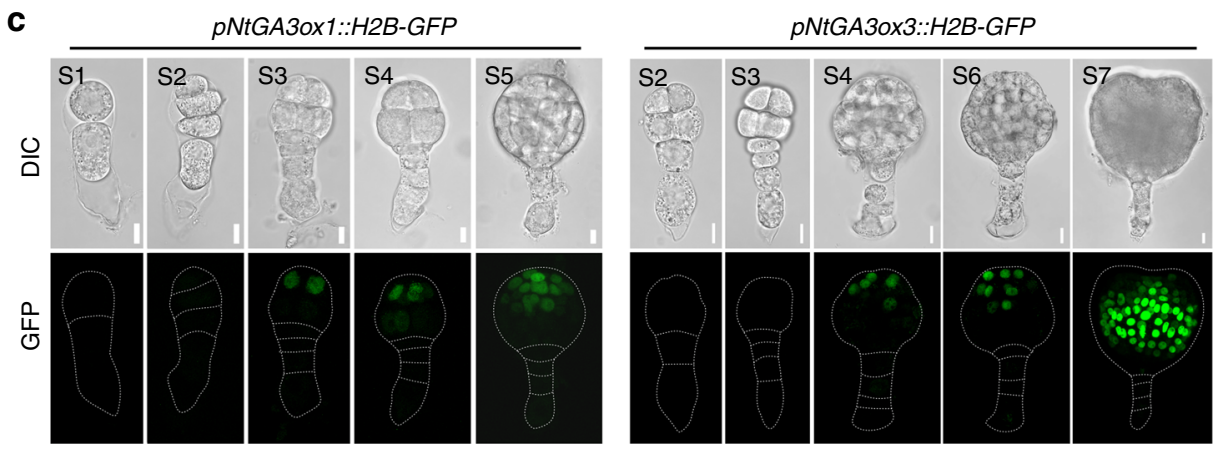

d

pNtGA3ox2::H2B-GFP
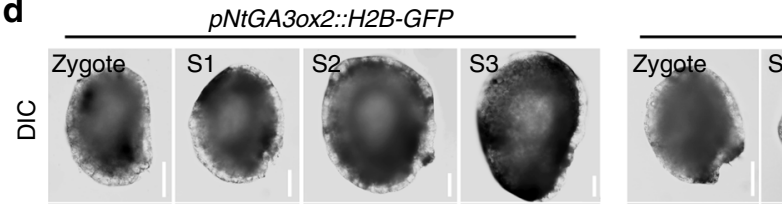

pNtGA3ox3::H2B-GFP
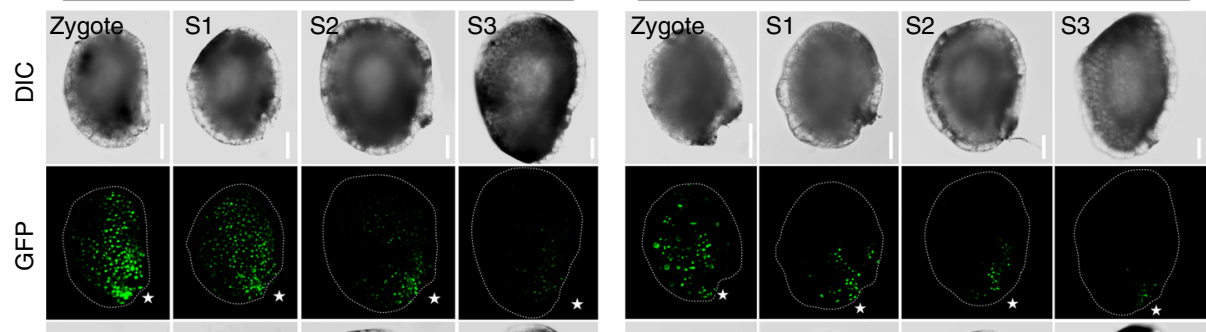

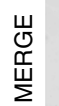
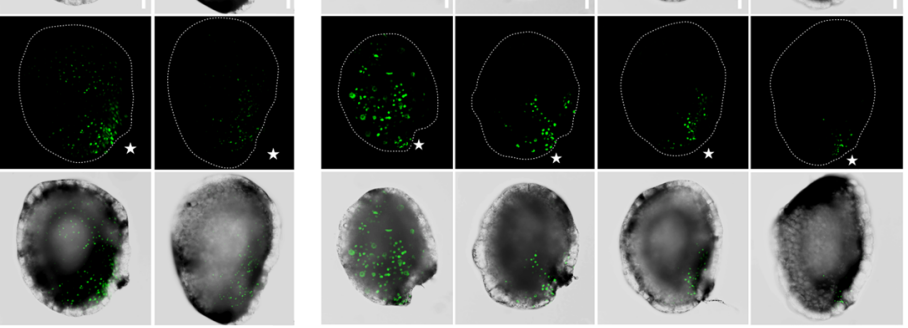

Fig. 4 Bioactive GA biosynthesis during early embryogenesis. a qRT-PCR analysis of NtGA3ox1, NtGA3ox2 and NtGA3ox3 expression; GAPDH was used as the internal control. b Expression of genes related to GA biosynthesis [NtGA200x1 (Ntc12, AB012856.1) and NtGA200x2 (Ntc16, AB016084.1)], deactivation [NtGA2ox1 (AB125232.1), NtGA2ox1-like (XM_016586923.1), NtGA2ox3 (EF471117), and NtGA2ox5 (EF471118)], and signaling in WT two-celled proembryos (stage 1) via transcriptome analysis, and via qRT-PCR analysis in WT four-celled proembryos (stage 2). GAPDH was used as the internal control. Values are means \pm SE of three biological replicates. nd, not detected. c Expression of NtGA3ox1 and NtGA3ox3 during embryogenesis. $\mathbf{d}$ Expression of NtGA3ox2 and NtGA3ox3 in the developing seed coat. Asterisks, micropylar ends; Scale bars: $10 \mu \mathrm{m}$ (c), $100 \mu \mathrm{m}$ (d). The source data of the graphs are provided in the Source Data file

occurs in the seed coat of Arabidopsis ${ }^{21}$. These data suggest that at least the location of GA biosynthesis in maternal tissue might be conserved during early embryogenesis. Thus, we further tested the influence of loss-of-DELLA function or deficient GA on the suspensor development in Arabidopsis. Indeed, the global-della mutant caused defected seeds and reduced fertility (Supplementary Fig. 13a), similar to that previously reported ${ }^{22}$. Moreover, we found that abnormal cell division was triggered in the suspensors of these abortive seeds (Supplementary Fig. 13b), suggesting that
loss-of-DELLA function promotes suspensor further development instead of PCD. For genetic evidence, we attempted to use WT pollen to fertilize the GA-deficient mutant gal-3 in Landsberg erecta (Ler) background. Unfortunately, ga1-3 mutant showed defective pistils (Supplementary Fig. 13c) and infertile ovules (Supplementary Fig. 13d), similar to that observed in ga1-3 in Columbia background ${ }^{23}, g a 1-1^{24}$, and ga20ox triple mutant ${ }^{23}$. As a result we were unable to conclusively analyze the maternal effects of GA on suspensor PCD in Arabidopsis. 
a
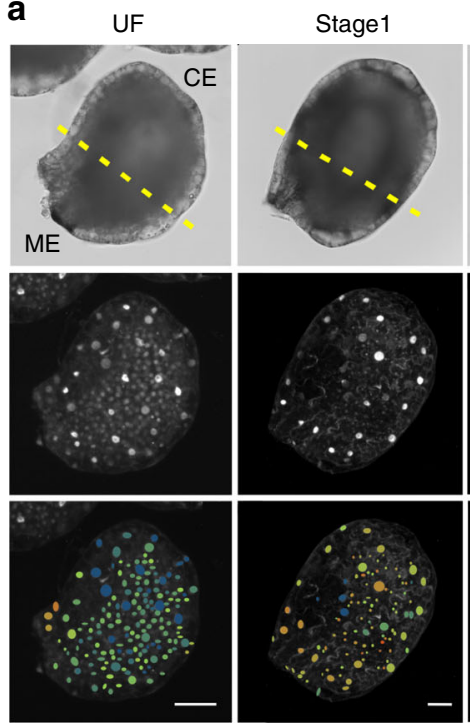

Stage1
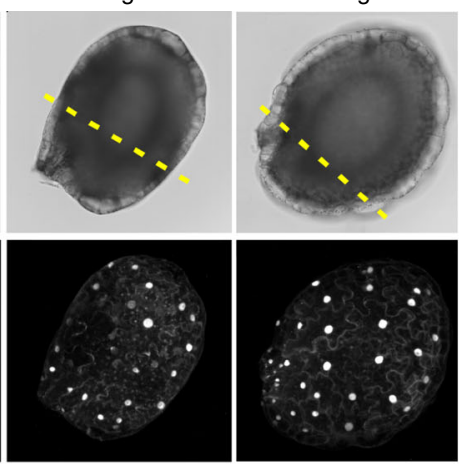

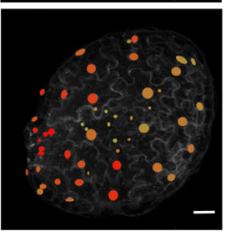

Stage3
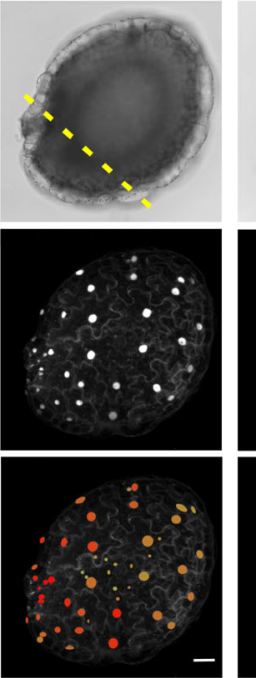
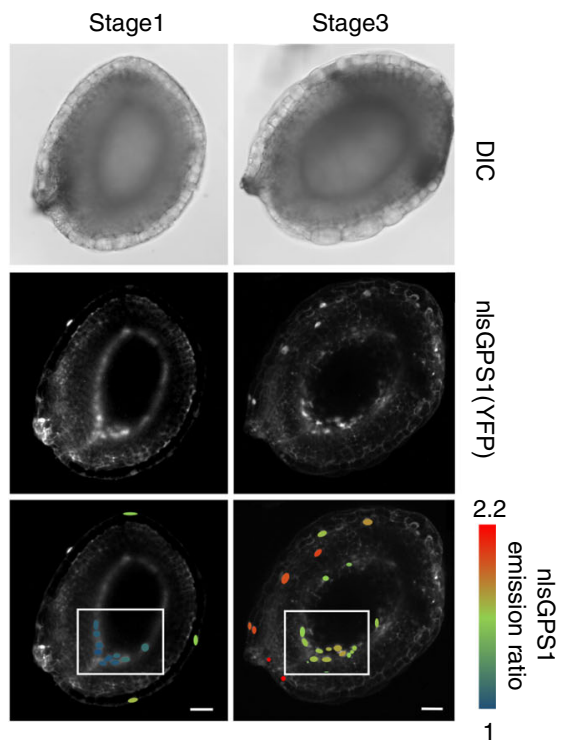
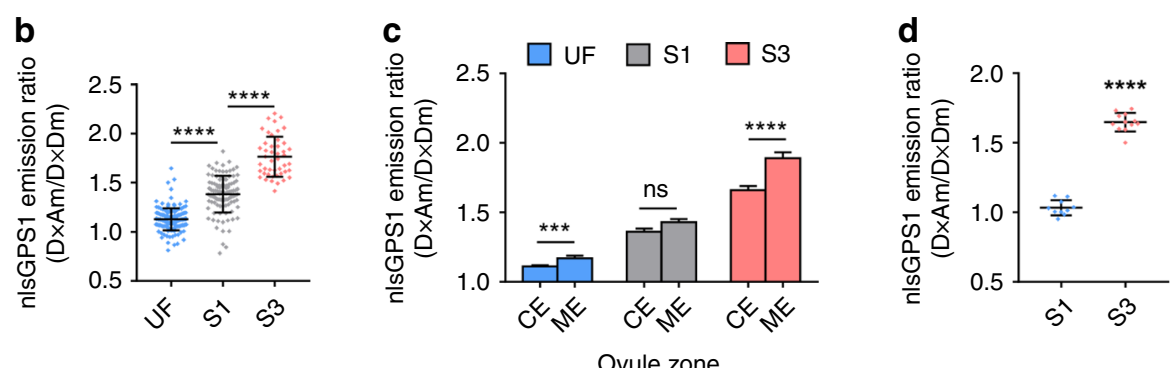

e
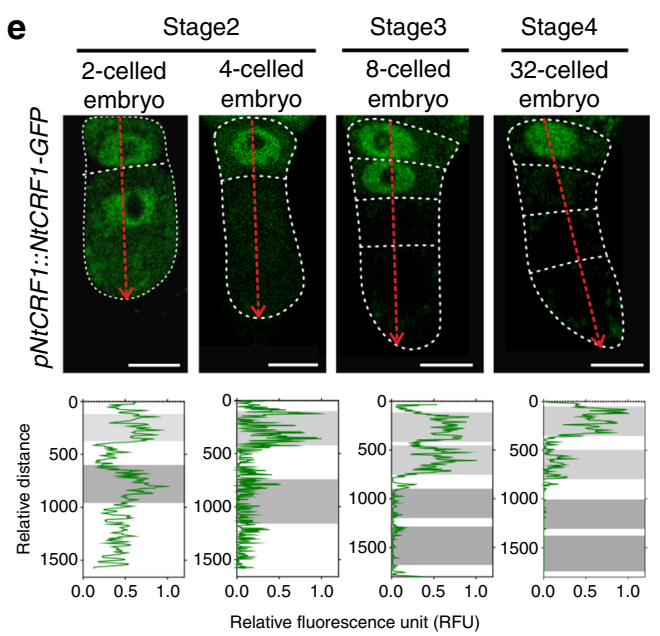
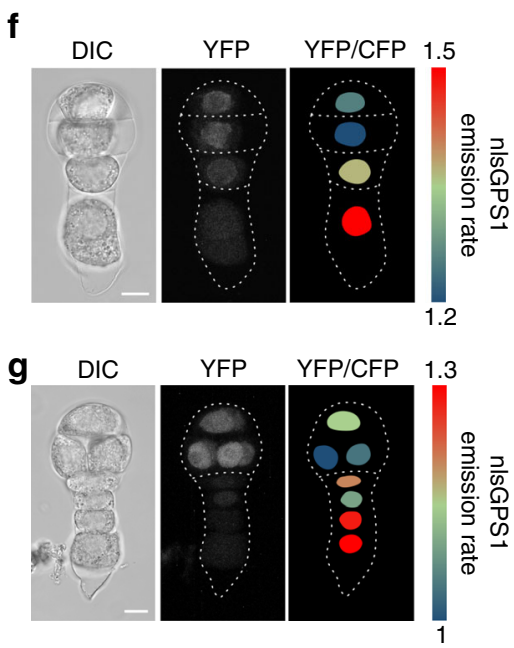

Fig. 5 Transient increase in, and polar distribution of, bioactive GA in seeds. a Left: Images of the nlsGPS1 emission ratios of unfertilized (UF), 4-DAP and 6DAP seeds. Right: Images of the nlsGPS1 emission ratios of an optical section of a 4-DAP or 6-DAP seed. Boxes: micropylar endothecium. $\mathbf{b}$ Box plot of the nlsGPS1 emission ratios of the whole seed coat $(n>46$ nuclei). c nlsGPS1 emission ratios of the micropylar and chalaza ends [dashed lines in $\mathbf{a} ; n>20$ nuclei]. $\mathbf{d}$ Box plot of the nlsGPS1 emission ratios of stage 1 or 3 seeds [boxed region in $\mathbf{a} ; n>10$ nuclei]. e NtCRF1-GFP was absent from the basal SC at stage 3. Fluorescence signal intensities along a line across all SCs (red arrows). X-axis, relative GFP signal intensity (green); $y$-axis, relative distances along the line. Gray shading, positions of nuclei. $\mathbf{f}$ and $\mathbf{g}$ Images of the nlsGPS1 emission ratios of 4-celled and 12-celled proembryos in WT background. Data are the means \pm SD (standard deviations) (Student's $t$-test; ns, $P>0.05$; ${ }^{\star \star \star} P<0.001 ;{ }^{\star \star \star \star} P<0.0001$ ). ME micropylar end, CE chalaza end. Scale bars: $100 \mu \mathrm{m}$ (a), $10 \mu \mathrm{m}(\mathbf{e}-\mathbf{g})$. The source data of the graphs are provided in the Source Data file

\section{Discussion}

In summary, we demonstrated that NtCRF1 binds to the M3 motif of the NtCYS promoter. In general, DELLA proteins do not contain classical DNA-binding domains, and thus require another $\mathrm{TF}$ to bind to promoter and modulate gene expression ${ }^{25,26}$. However, we found that NtCRF1 that has characteristics typical of DELLA proteins in response to GA can bind to the NtCYS promoter. Recently, a crystal structure analysis revealed that the GRAS domain of OsSCL7 binds to DNA in a dimer form ${ }^{27}$. We also found that NtCRF1 possesses both DELLA and GRAS domains (Supplementary Fig. 6b), and NtCRF1 and NtCRF2 could form a homo-dimer or 
a

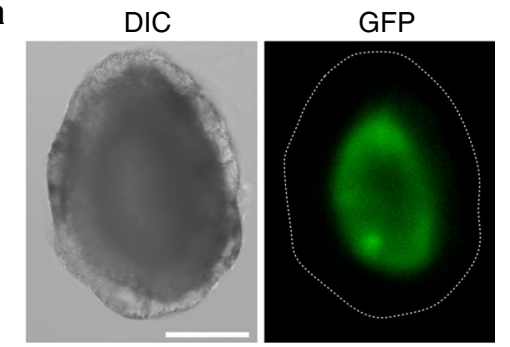

b
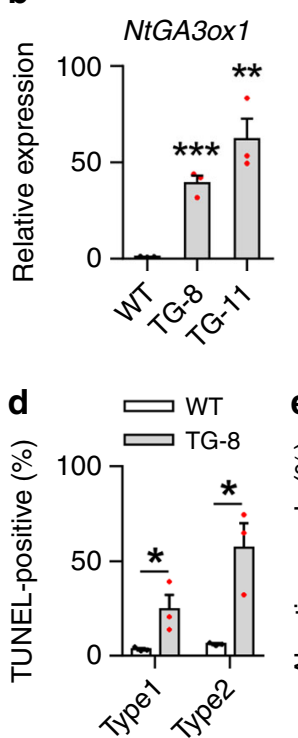

f

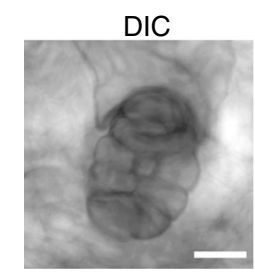

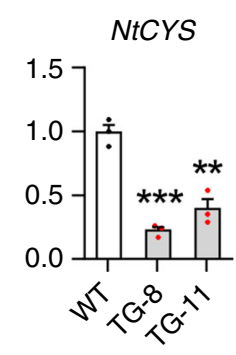

e

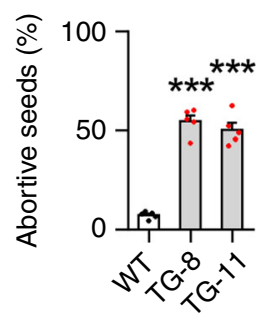

$\mathrm{Pl}$

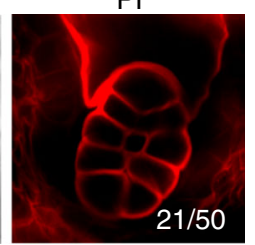

C

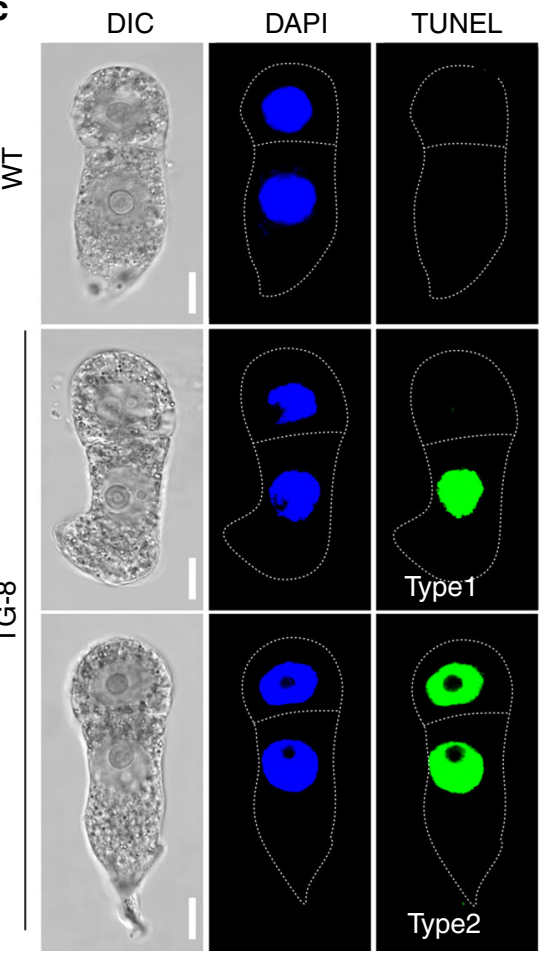

9
5
3
$\times$
0
0
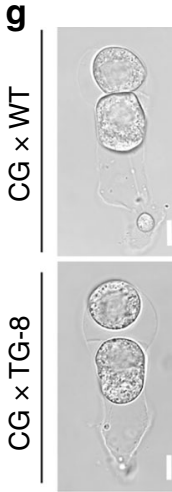
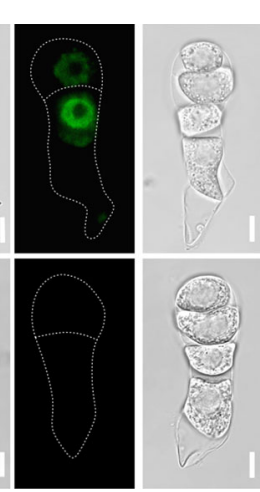

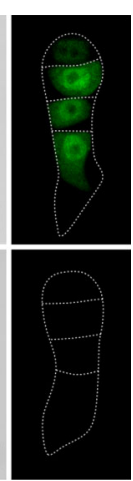

Fig. 6 Ectopic expression of NtGA3ox1-GFP in the endothelium triggers PCD. a Localization of NtGA3ox1-GFP in the endothelium of pNtTPE8::NtGA3ox1-GFP plant. $\mathbf{b}$ Increased expression of NtGA3ox1 and decreased expression of NtCYS in the TG-8 and TG-11 lines. c TUNEL test in two-celled proembryos from WT and TG-8. d Frequency of two-celled proembryos with TUNEL-positive apical and basal cells in WT and TG-8 $(n=105-112)$. e Frequency of aborted seeds in WT and TG-8. ( $n=1000-1500)$. f Pl-staining of abnormal embryos with no suspensor in the TG-8 line. $\mathbf{g}$ NtCRF1-GFP signal in early proembryos after crossing WT and pNtCRF1::NtCRF1-GFP (CG) lines. Data are the means \pm SE of 3 independent experiments in (b, c) and 5 in e (Student's $t$-test, ${ }^{\star} P<$ $\left.0.05,{ }^{\star \star} P<0.01,{ }^{\star \star \star} P<0.001\right)$; Scale bars: $150 \mu \mathrm{m}(\mathbf{a}), 10 \mu \mathrm{m}(\mathbf{c}, \mathbf{g}), 20 \mu \mathrm{m}(\mathbf{f})$. The source data of the graphs are provided in the Source Data file

hetero-dimer by $\mathrm{Y} 2 \mathrm{H}$ and that the GRAS domain of NtCRF1 may mediate its binding to the promoter of NtCYS (Supplementary Fig. 1b, g) as AtSHR and AtSCR interact directly with the promoters ${ }^{28}$. Moreover, we confirmed that NtCRF1 promotes the expression of NtCYS, and degradation of the former at stage 3 reduces NtCYS expression, resulting in the activation of $\mathrm{NtCP} 14$ and thence suspensor PCD. Thus, maternal GA signal and proembryo responsor NtCRF1 mediate maternal-proembryo communication via the GA-GID1-CRF1CYS-CP14 signaling cascade to precisely control the maternalembryo connection (Supplementary Fig. 14).

It was reported that GA could trigger tapetum PCD in flowering plants via the GAMYB transcription factor ${ }^{29}$, which is involved in reproductive development during land plant evolu$\operatorname{tion}^{30}$. Interestingly, we also found seven GAMYB-like genes expressed in tobacco two-celled proembryos, and thus, it is worthy to investigate the relationship between GAMYB and suspensor PCD in the near future. In fact, during land-plant evolution, the GA-DELLA regulatory mechanism arose via independent stepwise recruitment of the GA-stimulated GID1DELLA interaction ${ }^{13}$. In earlier stages [ 430 million years ago (MYA)], GA was not involved in the GID1-DELLA interaction. Later, GID1-DELLA interactions became susceptible to potentiation by bioactive GA during the period of the bryophyte and lycophyte divergences ( 430-400 MYA $)^{13}$. Notably, an embryonic suspensor-like structure was first generated in low plants during the same period $^{8}$, indicating that during plant evolution the establishment of the GA-dependent GID1-DELLA interaction was coupled to that of the suspensor structure. Thus, GA signaling is likely involved in maternal tissue-to-proembryo communication as it evolved in lower plants and maternal control of the connection between maternal tissue and the embryo might be an inborn character of embryogenesis for precise control of the duration of auxin and nutrient supply, which is critical for the 
embryos to establish full ability for independent development before their detachment from mother plant body.

Methods
Materials. Arabidopsis plants were grown in $16 \mathrm{~h}$ light $/ 8 \mathrm{~h}$ dark conditions at $20-22{ }^{\circ} \mathrm{C}$. N. tabacum L. cv. Petite Havana SR1 tobacco plants were grown under a $16 / 8 \mathrm{~h} \mathrm{light/dark}$ cycle at $25^{\circ} \mathrm{C}$. Ntcrf mutants were generated using the clustered regularly interspaced short palindromic repeats (CRISPR)/CRISPR-associated protein 9 (Cas9) system ${ }^{31,32}$. The CRISPR target of NtCRF was selected as described $^{31,32}$, and the vectors were transferred into SR1. The genomic region surrounding the CRISPR target sites of NtCRF were amplified by PCR, and the segment was subjected to Sanger sequencing to screen for mutants. T2 plants were used for phenotyping and T-DNA was already separated away, and identified using CRISPR/Cas9 vector-specific primers. Primer sequences are listed in Supplementary Data 1.

Screening for NtCYS regulative factors. Based on our previous work on transcript profiling of two-celled proembryo, we first screened all the TFs expressed in the two-celled proembryos. We found 1267 candidates that belong to 35 gene families. Then, we analyzed the promoter sequence of NtCYS via PlantPan ${ }^{11}$. We found five AATTT motifs in NtCYS promoter according to the report that GRAS could bind to the AATTT motif ${ }^{12}$. Thus, we only focused on GRAS family that expressed in the two-celled proembryos. We isolated these five motifs embedded within a 25-bp region of the surrounding promoter sequence according to the required length of a bait for $\mathrm{Y}_{1} \mathrm{H}^{12}$.

Yeast one-hybrid assays. Yeast one-hybrid assays were conducted using the Matchmaker One-Hybrid System (Clontech). The 25-bp AATTT region surrounding the NtCYS promoter sequences was synthesized with EcoRI and XbaI flanking restriction sites, and cloned into an EcoRI-XbaI-digested pHISi-1 bait vector. For expression in yeast, $N t C R F 1, N t C R F 2$, and other TF cDNAs were cloned into the pGAD424 prey vector. Saccharomyces cerevisiae YM4271 (Clontech) was used as the host. Transformation was confirmed by growth on SD medium containing $0,15,30$, or $60 \mathrm{mM} 3-\mathrm{AT}$ (a competitive inhibitor of the His 3 gene product) (A8056; Sigma). The primers used are shown in Supplementary Data 1.

Yeast two-hybrid assays. Yeast two-hybrid assays were performed according to the manufacturer's protocol (Clontech). Briefly, S. cerevisiae strain AH109 was transformed with the bait construct pGBKT7-NtGID1s and subsequently with pGADT7-NtCRF1. Vectors lacking coding region insertions were used as negative controls. Protein interactions were scored based on growth on defined synthetic medium lacking Trp, Leu, His, and adenine in the presence or absence of $10 \mu \mathrm{M}$ $\mathrm{GA}_{1}$ (G377495; TRC) and $\mathrm{GA}_{4}$ (G7276; Sigma). The primers used are shown in Supplementary Data 1.

ChIP-qPCR analysis. Pooled unfertilized ovaries $(\sim 1 \mathrm{~g})$ from $p N t C R F 1:: N t C R F 1$ GFP and $p N t C R F 1:: G F P$ plants were cross-linked under vacuum in $1 \%(\mathrm{w} / \mathrm{v})$ formaldehyde for $30 \mathrm{~min}$ at room temperature. Cross-linking was quenched by the addition of $0.125 \mathrm{M}$ glycine and vacuum infiltration continued for a further $15 \mathrm{~min}$. The cross-linked tissues were frozen in liquid nitrogen and ground to a fine powder. ChIP assays were performed using the ChIP Kit-Plants (ab117137; Abcam). Nuclei were extracted and chromatin was disrupted using an Ultrasonic Cell Disruptor (JY92-IIN; Scientz) with 10 cycles on ice (15 s each at $30 \%$ duty cycle/1 min cooling). Chromatin $(150 \mu \mathrm{l})$ was immunoprecipitated with $4 \mu \mathrm{l}$ of an anti-GFP antibody (ab290; Abcam) for $90 \mathrm{~min}$. The purified DNA fragments were used as the template for qPCR. qPCR was performed using the FastStart Essential DNA Green Master Mix (22682700; Roche) with three technical replicates, and threshold cycle numbers $(\mathrm{Ct})$ were determined using a CFX Connect Real-Time PCR Detection System (Bio-Rad). Fold enrichment of the targeted genomic sequences over IgG was calculated as: $\left.2{ }^{-\left(\mathrm{Ct}_{\mathrm{CHIP}}\right.}-\mathrm{Ct}_{\mathrm{IgG}}\right)$, where $\mathrm{Ct}_{\mathrm{ChIP}}$ and $\mathrm{Ct}_{\mathrm{IgG}}$ are the mean threshold cycles of triplicate PCRs of DNA samples immunoprecipitated using an anti-GFP antibody and the control IgG, respectively. All qPCR reactions were subjected to melt curve analyses and agarose gel electrophoresis to confirm the presence of a single specific product. The qPCR primer sequences are listed in Supplementary Data 1.

Electrophoretic mobility shift assay. The DNA-interaction domain of NtCRF1 (153-396 aa) and NtCRF2 (207-417 aa) was inserted into pET32a (Novagen), respectively. The resulting plasmid was transformed into Escherichia coli BL21 (DE3). The recombinant NtCRF1-His protein was expressed and purified according to the manufacturer's instructions. The 55-bp region of the promoter sequence surrounding AATTT was synthesized and the $5^{\prime}$-end was biotinylated (GenScript) and dissolved in a buffer (10 mM Tris and $1 \mathrm{mM}$ EDTA). EMSAs were performed using the LightShift Chemiluminescent EMSA Kit (20148; Thermofisher). The labeled oligonucleotides were added to a $20-\mu \mathrm{l}$ reaction mixture composed of 1-4 $\mu \mathrm{g}$ of the protein extract, $20 \mathrm{fmol}$ biotin end-labeled target DNA, 4 pmol unlabeled target DNA (competitor), $1 \times$ DNA binding buffer, $1 \mu \mathrm{g}$ poly(dI$\mathrm{dC}), 2.5 \%$ glycerol, $0.05 \% \mathrm{NP}-40,50 \mathrm{mM} \mathrm{KCl}$, and $5 \mathrm{mM} \mathrm{MgCl}_{2}$. After incubation for $20 \mathrm{~min}$ at $25^{\circ} \mathrm{C}$, the reaction products were resolved in $6 \%$ native polyacrylamide gels with $0.5 \times$ TBE buffer. The blots were developed using the Chemiluminescent Nucleic Acid Detection Module Kit (89880; Thermo Scientific). The primers used are shown in Supplementary Data 1.

Transient transcription dual-luciferase assay. The reporters were constructed based on pGreenII 0800-LUC vector ${ }^{33}$ and the effectors were constructed based on the pGreenII 62-SK vector. NtCYS promoter and the mutant promoter were cloned into the SalI and NcoI site of the pGreenII 0800-LUC vector, respectively. The full length of $N t C R F 1$ CDS was cloned into the XbaI and $K p n I$ site of the pGreenII 62SK vector. The protoplasts were isolated from mesophyll cells of $N$. benthamiana. The plasmids were transfected into the protoplasts followed by $16 \mathrm{~h}$ incubation in the dark at $25^{\circ} \mathrm{C}$. The enzyme activity was measured using Dual-Luciferase ${ }^{\oplus}$ Reporter Assay System (E1910; Promega) in a microplate reader Biotek ${ }^{\circledR}$ Cytation3. Four independent measurements were carried out for the analysis.

Quantification of endogenous GAs. For determination of endogenous gibberellin in the seeds at stage $-1,-3$, and -5 , we collected and grinded $500 \mathrm{mg}$ seeds into powder as one sample after quick freezing with liquid nitrogen. Each sample was extracted with $5 \mathrm{ml}$ of $90 \%$ aqueous methanol $(\mathrm{MeOH})$ and $2 \mathrm{ng}$ of each D-labeled GA compound as internal standards. The MAX cartridge (Waters) was activated and equilibrated with $10 \mathrm{ml} \mathrm{MeOH}$, water, $5 \% \mathrm{NH}_{4} \mathrm{OH}$, and $90 \% \mathrm{MeOH}$ in turn, while MCX (Waters) with $10 \mathrm{ml} \mathrm{MeOH}$, water and $90 \% \mathrm{MeOH}$ in order. Then the MAX cartridge was disconnected and washed with $5 \% \mathrm{NH}_{4} \mathrm{OH}$ in $5 \% \mathrm{MeOH}$, $\mathrm{MeOH}$ successively. Finally, GAs were eluted with $2 \%$ formic acid in $90 \% \mathrm{MeOH}$ After dried with $\mathrm{N}_{2}$ stream, the eluent was reconstructed with $200 \mu \mathrm{l} 80 \% \mathrm{MeOH}$ and subjected to UPLC-MS/MS analysis. GAs analysis was performed on a quadrupole linear ion trap hybrid mass spectrometer (QTRAP 5500, AB SCIEX) equipped with an electrospray ionization source coupled with a UPLC (Waters). Five microliter of each sample was injected into a BEH C18 column. GAs were detected in negative multiple reaction monitoring (MRM) mode. Each GA compound was quantified with a MRM transition and qualified with another one. Three independent samples were analyzed for each stage.

Identification of NtGA3ox genes. We firstly found GA3ox3 according the reference Ntab-TN90 Primary Assembly (BioProject: PRJNA319578) and its GO (Gene Ontology) annotation is 'gibberellin 3-beta-dioxygenase 4-like'. Except two known GA3oxs, we found five annotated GA3ox-like genes in N. tabacum (Supplementary Fig. 11a). By sequence analysis, we chose the four of them for further analysis since the sequence of another one is distinctive compared to GA3oxs (Supplementary Fig. 11b). We tested the six GA3oxs and found three of them (NtGA3ox-1, -2 , and -3) expressed in seeds before suspensor PCD. Thus, NtGA3ox-1, -2, and -3 were used for further analysis.

Embryo isolation and seed clearing. For embryo isolation, seeds were first treated in enzyme solution ( $1 \%$ cellulase R10, 0.5\% macerozyme R10, 10.5\% mannitol, and $3 \mathrm{mM}$ MES, pH 5.5) for $30 \mathrm{~min}$ in the dark. Then droplets of the seed suspension were gently grinded by a flat-headed glass rod. After grinding several droplets of $10.5 \%$ mannitol were added for releasing and washing embryo sacs. Living embryos could be further isolated by a second enzymatic maceration procedure followed by a brief micromanipulation.

For seed clearing, whole seeds were collected in a $2 \mathrm{ml}$-centrifuge tube and were fixed by $50 \%$ methanol and $10 \%$ acetic acid at $4{ }^{\circ} \mathrm{C}$ for 12 -h and then subjected to an overnight treatment of $1 \%$ SDS and $0.2 \mathrm{M} \mathrm{NaOH}$ at room temperature. Thereafter, the seeds were rinsed in water, followed by an incubation in $2.5 \%$ $\mathrm{NaClO}$ for 1-h, and then rinsed again. Next, the seeds were transferred into $1 \%$ periodic acid for 1 -h at room temperature and in $80 \%$ ethanol for $10 \mathrm{~min}$ at $80^{\circ} \mathrm{C}$ Subsequently, the seeds were transferred back to the same fixative and incubated for 1-h before the seeds were rinsed again with water. After washing, the seeds were incubated in Schiff reagent with propidium iodide (PI) (P4170; Sigma) $(100 \mathrm{mM}$ sodium metabisulphite and $0.15 \mathrm{M} \mathrm{HCl}$; PI to a final concentration of $0.1 \mathrm{mg} / \mathrm{ml}$ ) for about 3-h. The samples were stored in a chloral hydrate solution (4 g chloral hydrate, $1 \mathrm{ml}$ glycerol, and $2 \mathrm{ml}$ water) and kept overnight at room temperature. Next, the chloral hydrate was replaced by Hoyer's solution ( $30 \mathrm{~g}$ gum arabic, $200 \mathrm{~g}$ chloral hydrate, $20 \mathrm{~g}$ glycerol, and $50 \mathrm{ml}$ water). The seeds were kept in the Hoyer's solution for at least 10 days before observation under a confocal microscope.

RNA-seq and data analysis. mRNA of isolated two-celled proembryos (40-50 embryos per sample) was directly extracted using Dynabeads mRNA DIRECT ${ }^{\mathrm{TM}}$ Micro Kit (Life Technology). cDNA preparation was performed using the SMARTer Ultra Low Input RNA Kit for Sequencing v3 (Clontech). Libraries were constructed using TruePrep ${ }^{\mathrm{TM}}$ DNA Library Prep Kit V2 for Illumina (Vazyme) and then sequenced on Illumina HiSeq ${ }^{\mathrm{TM}}$ 4000. Adapters and low-quality reads were filtered using Cutadapt and an in-house script. Next, clean reads were mapped to the tobacco genome (N. tabacum genome reference, Ntab-TN90) using Bowtie 2. Gene expression levels were quantified as FPKM (fragments per kilobase of transcript per million mapped reads) using RSEM. The raw data for each sample are available in the NCBI Gene Expression Omnibus under accession number GSE133373. 
Cell viability and DNA fragmentation analyses. To evaluate cell viability and plasma membrane integrity, we stained embryos with fluorescein diacetate (FDA) (F7378; Sigma) and PI. Isolated embryos were incubated in the solution containing $10.5 \%$ mannitol, $2 \mu \mathrm{g} / \mathrm{ml} \mathrm{FDA}$, and $1 \mu \mathrm{g} / \mathrm{ml} \mathrm{PI}$ for $15 \mathrm{~min}$ at room temperature and washed twice with $10.5 \%$ mannitol before observation. To detect nuclear DNA fragmentation, the isolated embryos were tested by DeadEnd ${ }^{\mathrm{TM}}$ Fluorometric TUNEL System (G3250; Promega) or In Situ Cell Death Detection Kit, TMR red (12156792910; Roche) for $p N t C R F 1:: N t C R F 1-G F P$ line according to the manufacturer's instructions, respectively.

Two-celled proembryo culture in vitro. The ovaries were rapidly surfacesterilized with $75 \%$ alcohol, followed by 5 -min washes in sterile water for three times. Seeds were then isolated from the ovaries and collected in $2 \mathrm{ml} 13 \%$ mannitol for co-culture. The isolated two-celled proembryos were transferred into a Millicell (PICM01250; Millipore) containing $100 \mu$ l of the medium ${ }^{16}$. The Millicell was put in a $3-\mathrm{cm}$ Petri dish containing $1.5 \mathrm{ml}$ of the medium ${ }^{16}$, and $100-150$ seeds were co-cultured as feeder at $25^{\circ} \mathrm{C}$ in the dark. $1 \mu \mathrm{M} \mathrm{GA}_{4}$ or $1 \mu \mathrm{M} \mathrm{ABA}$ (A1049; Sigma) were added to the medium as appropriate.

PAC, GA, ABA, and ethyl alcohol treatment assays. Two-celled or four-celled proembryo isolated form $p N t C R F 1:: N t C R F 1-G F P$ line and treatment with $100 \mu \mathrm{M}$ $\mathrm{GA}_{4}$ for hours, $0.05 \%$ ethyl alcohol as control. Embryos derived from in cultured seeds form $p N t C R F 1:: N t C R F 1-G F P$ line after 72-h treatment with $100 \mu \mathrm{M}$ PAC, $0.05 \%$ ethyl alcohol as control. 4-DAP WT seeds treated with $100 \mu \mathrm{M} \mathrm{ABA}, \mathrm{PAC}$ (46046; Sigma), or $\mathrm{GA}_{4}$ for 12 -h, $0.05 \%$ ethyl alcohol as control, then collected for qRT-PCR.

Quantitative real-time PCR. Total RNA was extracted from leaves, roots, stems, anthers, sepals, and seeds using the MiniBEST Plant RNA Extraction Kit (9769; Takara). Total RNAs were treated with RNase-free DNase I (Promega), and cDNA was synthesized using Transcriptor Reverse Transcriptase (Invitrogen) according to the manufacturer's instructions. $0.2 \mu \mathrm{l}$ of resulting first-strand cDNA was used as a PCR template for qRT-PCR. Quantitative PCR analysis were performed using FastStart Universal SYBR Green Master (4913850001; Roche) with a CFX Connect ${ }^{\mathrm{TM}}$ Real-Time System (Bio-Rad). Glyceraldehyde-3-phosphate dehydrogenase (GAPDH), or both GAPDH and Ubiquitin-conjugating enzyme2 (UBI) were used as the internal reference(s) to normalize the relative level of each transcript. Each experiment was repeated three or five times and each time the experiment included duplicate samples. The qPCR primer sequences are listed in Supplementary Data 1. The raw data are shown in Source Data file.

Transformation of BY-2 cells. About $4 \mathrm{ml}$ of 1 -week-old tobacco BY- 2 culture was first transferred into a $60-\mathrm{mm} \times 15$-mm Petri dish, and then $20 \mu \mathrm{l}$ prepared Agrobacterium tumefaciens line GV3101, containing $p 35 S:: N t C R F 1-G F P$ or $p 35 S::$ (crf1- 117 )-GFP, was added into the culture dishes, respectively. Then each plate was sealed with Parafilm and incubated for 3-day at $28^{\circ} \mathrm{C}$ in the dark on a shaker at 100 r.p.m. Subsequently, the cultured cells were washed four times and dipped in NT1 medium ${ }^{34}$ containing $250 \mathrm{mg} / \mathrm{L}$ carbenicillin for $30 \mathrm{~min}$ to kill Agrobacterium, and then plated onto solid NT1 medium containing $250 \mathrm{mg} / \mathrm{L}$ carbenicillin and $50 \mathrm{mg} / \mathrm{L}$ kalamycin. After about 30-day, kalamycin-resistant calli appeared on the medium, and subsequently they were transferred onto new plates and suspended again in $50 \mathrm{ml}$ liquid NT1 medium for observation. The cells were treated with $100 \mu \mathrm{M} \mathrm{GA}_{3}$ for 4 -h and $0.05 \%$ ethyl alcohol as control. The primers used are shown in Supplementary Data 1.

Confocal microscopy and image analysis. Stained embryos were observed under a confocal microscope (Leica SP8). The nlsGPS1 emission ratio was determined as described $^{18}$. Images were processed using ImageJ or Adobe Photoshop CC.

Quantification and statistical analysis. The fluorescence intensity was calculated as: integrated density - (area of selected cell nucleus $\times$ mean background fluorescence). qRT-PCR and fluorescence intensity data were assumed to follow normal distributions and were subjected to Student's $t$-test or analysis of variance (ANOVA) using GraphPad Prism 7 software. The value of $n$, the definition of the center, dispersion, precision, and statistical significance are provided in the figure legends. ${ }^{\star} P<0.05 ;{ }^{\star \star} P<0.01 ;{ }^{* *} P<0.001 ;{ }^{* * * *} P<0.0001$ compared to mock controls, unless otherwise specified by connecting lines.

Reporting summary. Further information on research design is available in the Nature Research Reporting Summary linked to this article.

\section{Data availability}

Data supporting the findings of this study are available within the main text and its Supplementary Information files. The source data underlying Figs. 1c-e, 1g, 2b, 2d, 2e, 2g, 2h, 3b, 3d, 3f-h, 4a, 4b, 5b-d, 5f, 5g, 6b, 6d, 6e, and Supplementary Figs. 1c-f, 2a, 3d, 3e, $4 \mathrm{c}, 4 \mathrm{e}, 4 \mathrm{~g}, 4 \mathrm{~h}, 5 \mathrm{a}, 5 \mathrm{c}, 8 \mathrm{c}, 8 \mathrm{e}, 8 \mathrm{f}, 8 \mathrm{~g}, 8 \mathrm{i}, 10 \mathrm{~b}, 12 \mathrm{~b}-\mathrm{d}$ are provided as a Source Data file. RNAseq data has been deposited at the NCBI Gene Expression Omnibus database under accession number GSE133373. All data are available from the corresponding author upon request.

Received: 13 December 2018 Accepted: 12 July 2019

Published online: 02 August 2019

\section{References}

1. Constancia, M. et al. Placental-specific IGF-II is a major modulator of placental and fetal growth. Nature 417, 945-948 (2002).

2. Bonnin, A. et al. A transient placental source of serotonin for the fetal forebrain. Nature 472, 347-350 (2011).

3. Perez-Garcia, V. et al. Placentation defects are highly prevalent in embryonic lethal mouse mutants. Nature 555, 463-468 (2018).

4. Vento-Tormo, R. et al. Single-cell reconstruction of the early maternal-fetal interface in humans. Nature 563, 347-353 (2018)

5. Friml, J. et al. Efflux-dependent auxin gradients establish the apical-basal axis of Arabidopsis. Nature 426, 147-153 (2003).

6. Liu, Y. et al. Direct evidence that suspensor cells have embryogenic potential that is suppressed by the embryo proper during normal embryogenesis. Proc. Natl Acad. Sci. USA 112, 12432 (2015).

7. Robert, H. S. et al. Maternal auxin supply contributes to early embryo patterning in Arabidopsis. Nat. Plants 4, 548-553 (2018).

8. Kawashima, T. \& Goldberg, R. B. The suspensor: not just suspending the embryo. Trends Plant Sci. 15, 23-30 (2010).

9. Yeung, E. C. \& Meinke, D. W. Embryogenesis in angiosperms: development of the suspensor. Plant Cell 5, 1371-1381 (1993).

10. Zhao, P. et al. A bipartite molecular module controls cell death activation in the Basal cell lineage of plant embryos. PLoS Biol. 11, e1001655 (2013).

11. Chang, W. et al. PlantPAN: plant promoter analysis navigator, for identifying combinatorial cis-regulatory elements with distance constraint in plant gene groups. BMC Genom. 9, 561 (2008).

12. Hirsch, S. et al. GRAS proteins form a DNA binding complex to induce gene expression during nodulation signaling in Medicago truncatula. Plant Cell 21, 545-557 (2009).

13. Yasumura, Y., Crumpton-Taylor, M., Fuentes, S. \& Harberd, N. P. Step-bystep acquisition of the gibberellin-DELLA growth-regulatory mechanism during land-plant evolution. Curr. Biol. 17, 1225-1230 (2007)

14. Steber, C. M. A role for the ubiquitin-26S-proteasome pathway in gibberellin signaling. Trends Plant Sci. 8, 492-497 (2003).

15. Sierro, N. et al. The tobacco genome sequence and its comparison with those of tomato and potato. Nat. Commun. 5, 3833-3833 (2014).

16. He, Y., He, Y., Qu, L., Sun, M. \& Yang, H. Tobacco zygotic embryogenesis in vitro: the original cell wall of the zygote is essential for maintenance of cell polarity, the apical-basal axis and typical suspensor formation. Plant J. 49 515-527 (2007).

17. $\mathrm{Hu}$, J. et al. Potential sites of bioactive gibberellin production during reproductive growth in Arabidopsis. Plant Cell 20, 320-336 (2008).

18. Rizza, A., Walia, A., Lanquar, V., Frommer, W. B. \& Jones, A. M. In vivo gibberellin gradients visualized in rapidly elongating tissues. Nat. Plants $\mathbf{3}$, 803-813 (2017).

19. Schmidt, G. W. \& Delaney, S. K. Stable internal reference genes for normalization of real-time RT-PCR in tobacco (Nicotiana tabacum) during development and abiotic stress. Mol. Genet. Genom. 283, 233-241 (2010).

20. Nodine, M. D. \& Bartel, D. P. Maternal and paternal genomes contribute equally to the transcriptome of early plant embryos. Nature 482, 94-97 (2012).

21. Figueiredo, D. D. et al. Auxin production in the endosperm drives seed coat development in Arabidopsis. eLife 5, e20542 (2016).

22. Gomez, M. D. et al. Gibberellins regulate ovule integument development by interfering with the transcription factor ATS. Plant Physiol. 172, 2403 (2016)

23. Plackett, A. R. G. et al. Analysis of the developmental roles of the Arabidopsis gibberellin 20-oxidases demonstrates that GA20ox1, -2, and -3 are the dominant paralogs. Plant Cell 24, 941-960 (2012).

24. Goto, N. \& Pharis, R. P. Role of gibberellins in the development of floral organs of the gibberellin-deficient mutant, ga1-1, of Arabidopsis thaliana. Can. J. Bot. 77, 944-954 (1999)

25. De, L. M. et al. A molecular framework for light and gibberellin control of cell elongation. Nature 451, 480-484 (2008).

26. Feng, S. et al. Coordinated regulation of Arabidopsis thaliana development by light and gibberellins. Nature 451, 475-479 (2008).

27. $\mathrm{Li}, \mathrm{S}$. et al. Crystal structure of the GRAS domain of SCARECROW-LIKE7 in Oryza sativa. Plant Cell 28, 1025-1034 (2016).

28. Cui, H. et al. An evolutionarily conserved mechanism delimiting SHR movement defines a single layer of endodermis in plants. Science 316, 421-425 (2007).

29. Aya, K. et al. Gibberellin modulates anther development in rice via the transcriptional regulation of GAMYB. Plant Cell 21, 1453 (2009). 
30. Aya, K. et al. The Gibberellin perception system evolved to regulate a preexisting GAMYB-mediated system during land plant evolution. Nat. Commun. 2, 544 (2011).

31. Gao, J. et al. CRISPR/Cas9-mediated targeted mutagenesis in Nicotiana tabacum. Plant Mol. Biol. 87, 99-110 (2015).

32. Ma, X. et al. A robust CRISPR/Cas9 system for convenient, high-efficiency multiplex genome editing in monocot and dicot plants. Mol. Plant $\mathbf{8}$, 1274-1284 (2015).

33. Hellens, R. P. et al. Transient expression vectors for functional genomics, quantification of promoter activity and RNA silencing in plants. Plant Methods 1, 13 (2005)

34. Mayo, K. J., Gonzales, B. J. \& Mason, H. S. Genetic transformation of tobacco NT1 cells with Agrobacterium tumefaciens. Nat. Protoc. 1, 1105-1111 (2006).

\section{Acknowledgements}

We thank Prof. Yao-Guang Liu (South China Agricultural University) for providing the CRISPR/Cas9 system, Dr. Xiangdong Fu (Institute of Genetics and Developmental Biology-CAS) for gal-3 seeds, Dr. Patrick Achard (CNRS-University) for global-della seeds, Prof. Genji Qin (Peking University) for the dual-luciferase assay system and Dr. Sheliang Wang (Huazhong Agricultural University) for BY-2 cells. We also appreciate the expertize of Dr. Peiyong Xin and Dr. Jinfang Chu (Institute of Genetics and Developmental Biology-CAS) in determining the GAs. National Natural Science Foundation of China Key Project (31630094), National Natural Science Foundation of China Project (31400171), and Young Elite Scientists Sponsorship Program by CAST (YESS) (2015QNRC001) support the work.

\section{Author contributions}

Conceptualization: M.S. and C.S.; methodology: C.S., P.L. and P.Z.; investigation: C.S. P.L., Y.D., H.C., X.H., T.C., A.L., H.L. and W.Y.; writing-original draft: C.S. and P.L.; writing-review and editing: M.S. Funding acquisition, M.S. and P.Z.; resources: M.S.

\section{Additional information}

Supplementary Information accompanies this paper at https://doi.org/10.1038/s41467019-11476-3.

Competing interests: The authors declare no competing interests.

Reprints and permission information is available online at http://npg.nature.com/ reprintsandpermissions/

Peer review information: Nature Communications thanks Dolf Weijers, David Weiss and other anonymous reviewer(s) for their contribution to the peer review of this work.

Publisher's note: Springer Nature remains neutral with regard to jurisdictional claims in published maps and institutional affiliations.

\section{(c) (i)} Commons license, and indicate if changes were made. The images or other third party material in this article are included in the article's Creative Commons license, unless indicated otherwise in a credit line to the material. If material is not included in the article's Creative Commons license and your intended use is not permitted by statutory regulation or exceeds the permitted use, you will need to obtain permission directly from the copyright holder. To view a copy of this license, visit http://creativecommons.org/ licenses/by/4.0/.

(C) The Author(s) 2019 